\title{
The social functional outcome of being naturalistically treated with paliperidone extended-release in patients with schizophrenia
}

This article was published in the following Dove Press journal:

Neuropsychiatric Disease and Treatment

22 June 2015

Number of times this article has been viewed

\section{Ryoko Nakagawa' \\ Takashi Ohnishi' \\ Hisanori Kobayashi ${ }^{1}$ \\ Akihide Wakamatsu ${ }^{2}$ \\ Ai Tanimura ${ }^{3}$ \\ Kazuo Morita ${ }^{3}$ \\ Toshio Yamaoka ${ }^{3}$ \\ Hideo Usui ${ }^{3}$ \\ Yoshimasa Ogawa ${ }^{3}$ \\ Akiko Fujino ${ }^{3}$ \\ Kazutake Yoshizawa'}

'Evidence Generation Department, Medical Affairs Division, ${ }^{2}$ Medical

Affairs Strategy Department, Medical

Affairs Division, ${ }^{3}$ Drug Safety

Surveillance Department, Japan Safety

and Surveillance Division, Janssen

Pharmaceutical K.K., Tokyo, Japan
Correspondence: Takashi Ohnishi Evidence Generation Department, Medical Affairs Division, Janssen

Pharmaceutical K.K., 5-2, Nishi-kanda 3-chome Chiyoda-ku, Tokyo I0I-0065, Japan

Tel +8I 344 II 5009

Fax +8I 3 44II 5086

Email tohnish8@its.jnj.com
Background: Social functioning is an important outcome for patients with schizophrenia. To evaluate the effects of paliperidone extended-release (PAL-ER) on social function, symptomatology, and safety in the routine clinical practice, we conducted a 1-year post-marketing surveillance study of PAL-ER. We also explored relationships between symptomatic improvement and socially functional outcome in patients with schizophrenia.

Patients and methods: Patients with an established diagnosis of schizophrenia were allowed flexible 3-12 mg/day dosing during the surveillance. Patients were assessed on social functioning using the Social and Occupational Functioning Assessment Scale (SOFAS) and on symptomatology using the Clinical Global Impression-Schizophrenia scale. All adverse events (AEs) were also collected.

Results: A total of 1,429 patients were enrolled in the surveillance study, of whom 1,405 were evaluable for safety and 1,142 were evaluable for efficacy. The treatment discontinuation rate for any reason during the observation period was 34.66\%. Significant improvements were observed on both Social and Occupational Functioning Assessment Scale and Clinical Global Impression-Schizophrenia scale during the observation period. The percentage of patients with socially functional remission (SOFAS $\geq 61$ ) also increased significantly. A significant association between early improvements in positive symptoms, sex, severity of negative symptoms at baseline, and socially functional remission was observed. A total of $33.52 \%$ of patients had AEs and $8.75 \%$ of patients had serious AEs. Despite the recommendation of monotherapy with PAL-ER, 65.84\% of patients were given additional antipsychotics (polypharmacy). Post hoc comparisons of monotherapy versus polypharmacy revealed that the monotherapy group had better outcomes and fewer AEs than the polypharmacy treated group. The improvement in social functioning and the rate of socially functional remission did not differ between groups.

Conclusion: PAL-ER treatment showed effective symptom control and improvement in social functioning. The data suggest that early response to antipsychotic treatment should be important for functional outcomes.

Keywords: paliperidone, social function, schizophrenia, naturalistic study

\section{Introduction}

Pharmacological treatment in schizophrenia has focused on the reduction in symptomatology, ie, mean change in score for specific psychiatric scales, such as Positive and Negative Symptom Scale, during a relatively short period (6-12 weeks) as evidence of successful treatment. Actually, the guideline on the clinical investigation of antipsychotics by the European Medical Agency mentioned that primary efficacy measures should be presented as the numerical change in schizophrenia symptom score from baseline to study end point. ${ }^{1}$ Nevertheless, the most important treatment goal in patients with schizophrenia 
is enabling each patient to re-engage in meaningful life experiences, namely functional remission and recovery from schizophrenia. ${ }^{2}$ Regarding symptomatic remission, the Remission in Schizophrenia Working Group has proposed their definition for remission; however, it requires further examination of validity and utility, as well as future refinement, particularly in relation to psychosocial functioning and cognitive dysfunctions associated with functional remission and recovery. ${ }^{3}$

Social function is one of the important functional outcomes for patients with schizophrenia. ${ }^{4-7}$ Several independent factors such as upbringing, premorbid personality and adjustment, social circumstance, shorter duration of untreated psychosis, etc contribute to functional outcomes. ${ }^{8-12}$ Although antipsychotics have been used primarily to control symptoms, particularly positive symptoms and not with any direct therapeutic effects on cognition, social function, or quality of life related to functional outcomes, improvement of functional outcomes including social function should be mediated by symptom control. ${ }^{13}$ Furthermore, several studies suggested that early symptom responses to antipsychotics should be associated with good functional outcomes, remission, and recovery. ${ }^{1-12,14-17}$

Paliperidone extended-release (PAL-ER) designed to deliver paliperidone, an active metabolite of risperidone, was approved in the United States, European Union, Japan, and many other countries for the treatment of schizophrenia. Previous studies have demonstrated the effective outcome of PAL-ER in terms of personal and social functioning improvement evaluated by the Personal and Social Performance (PSP) scale as well as symptomatic improvement. ${ }^{18-22}$ The majority of these studies were randomized, controlled, shorter in duration, and have studied the effects of fixed doses in selected and homogeneous groups of patients. Therefore, evidence about the treatment effects on social function in routine clinical practice has been limited.

We conducted a 1-year post marketing surveillance of PAL-ER to evaluate effects on social function, symptomatology, and safety in a naturalistic setting. The aims of the present study are:

1. To demonstrate improvement of social function evaluated by Social and Occupational Functioning Assessment Scale (SOFAS) ${ }^{23}$ and symptomatology evaluated by Clinical Global Impression-Schizophrenia (CGI-SCH) scale, ${ }^{24}$ as well as time to discontinuation during 1-year treatment with PAL-ER.

2. To assess safety of PAL-ER in clinical practice.

3. To clarify the relationship between improvement of symptomatology and the social functional outcome: association between early symptom responses to PAL-ER and social functional outcome and which domain of improvement of symptomatology, ie, CGI-SCH negative, contributes to social functional outcome.

\section{Patients and methods Study design}

This was a 12-month, open-label, observational, multicenter surveillance study on the use of PAL-ER, newly initiated as antipsychotic treatment. Patients meeting selection criteria were men and women with an established diagnosis of schizophrenia, who had recently been switched to, or started on, PAL-ER. Patients having a history of allergic reactions to risperidone were excluded. Physicians were advised that all treatments and dose adjustments should be based on approved local labels, and that management decisions should be made at the physician's discretion, using clinical judgment and routine practice. In general, treatment was recommended to be started with $6 \mathrm{mg}$ PAL-ER daily. Flexible maintenance dosing with PAL-ER 3-12 mg/day was recommended, with dose adjustment indicated by the physician's assessment based on individual clinical response and tolerability. No study drug was provided: patients received medication according to their usual care. The protocol was reviewed by internal review board members including the ethical point of view and was approved by the Pharmaceuticals and Medical Devices Agency.

\section{Assessments}

Symptom severity was assessed with the CGI-SCH ${ }^{24}$ at baseline, 2 weeks, 4 weeks, 6 weeks, 8 weeks, 12 weeks, 6 months, 9 months, and 12 months. CGI-SCH was scored on a 7-point scale from $1=$ "normal" to $7=$ "among the most extremely ill" for each symptom domain (overall, positive, negative, depressive, cognitive). Social function was assessed with SOFAS, developed by the American Psychiatric Association for the Diagnostic and Statistical Manual of Mental Disorders, Fourth Edition (DSM-IV) to operationalize functioning, which was improved on the Global Assessment of Functioning Scale by incorporating the impact of psychological and general medical symptoms on patient functioning. ${ }^{23}$ The SOFAS was scored on a scale of $0-100$ as a 10-point scale from 1-10 as " $1 "=$ "Persistent hygiene problems" to $91-100$ as " $10 "=$ "Superior functioning" for the current period at baseline, 3 months, 6 months, and 12 months. Social functional remission was defined as SOFAS score $\geq 61$ points at 12 months. All AEs were also collected.

Electronic Data Capture system was used and the majority of the data were transcribed by the physicians from the source documents on to the electronic case report form and then transmitted in a secure manner to the sponsor. 


\section{Statistical analyses}

Although we recommended PAL-ER monotherapy, more than $65 \%$ of patients were given other antipsychotics combined with PAL-ER (polypharmacy); therefore, we performed post hoc analyses for evaluating the differences between monotherapy and polypharmacy groups (monotherapy versus polypharmacy).

The discontinuation rate was calculated by the KaplanMeier method, and the monotherapy versus polypharmacy comparison in discontinuation rate was performed using the log-rank test. Time effects on SOFAS and CGI-SCH scores were analyzed by generalized linear mixed models (GLMM). The interaction of time $\times$ monotherapy versus polypharmacy, age, sex, SOFAS, and CGI-SCH scores at the baseline were modeled. The last observation carried forward approach was used to handle missing data. Post hoc comparisons for monotherapy versus polypharmacy following GLMM were done with $t$-tests for each time point against baseline using Bonferroni adjustment. Regarding the social functional remission, factors which are associated with the social functional remission and changes in the rate of the social functional remission between the baseline and the end of the study were analyzed. To identify variables that explain social functional remission, logistic regression analysis was performed. Variance inflation factor was used to check for multicollinearity. The factors associated with social functional remission were regressed on delta (follow-up minus baseline) or baseline CGI-SCH scores and patient's demographic characteristics such as sex, age, and monotherapy versus polypharmacy. A GLMM was applied to examine the association of social functional remission and the interaction of time $\times$ monotherapy versus polypharmacy. The Wilcoxon rank sum test, the Fisher's exact test, and the chi-square test were used to assess comparisons between demographic and the incidence of AEs for monotherapy versus polypharmacy. Two-sided $P$-values less than 0.05 were considered statistically significant. Statistical analyses were conducted using R Statistical Software version 3.1.0 (Foundation for Statistical Computing, Vienna, Austria) and SAS version 9.3 (SAS Institute Inc., Cary, NC, USA). GLMM was performed with the GLIMMIX procedures of SAS.

\section{Results}

\section{Baseline demographics and clinical characteristics}

A total of 1,429 patients were enrolled in the surveillance study, of whom 1,405 were evaluable for safety and 1,142 were evaluable for efficacy (Figure 1). Two hundred and forty-five subjects who were coded as "incomplete for efficacy evaluation" by physicians were removed from efficacy dataset because of the lack of baseline information. Patient characteristics of each analytical group are summarized in Table 1. Baseline characteristics appeared similar in both analytical groups. The mean \pm standard deviation (SD) age was $46.67 \pm 15.45$ years and $51.06 \%$ of patients were female. About $30.83 \%$ of patients were hospitalized at study entry. The mean \pm SD daily dose of PAL-ER was

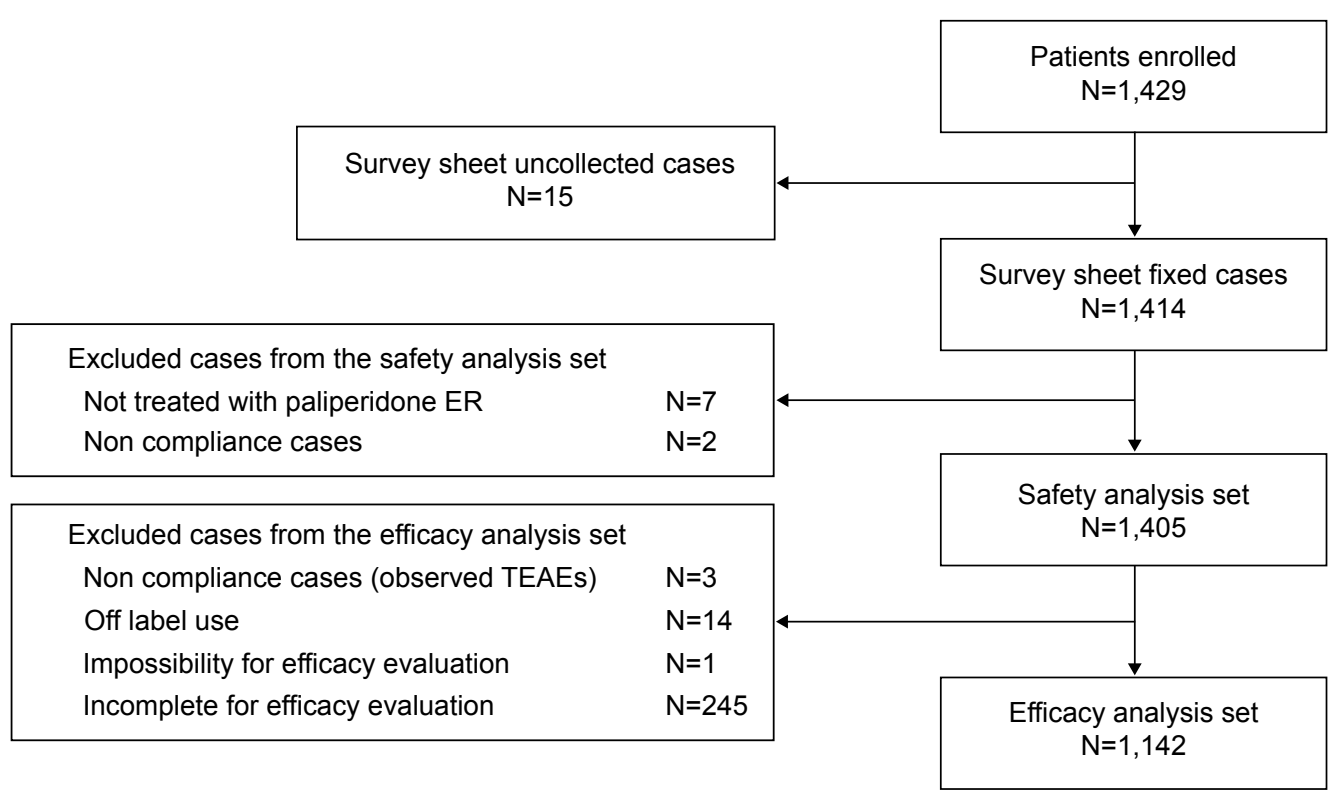

Figure I Subject flowchart of analysis.

Abbreviations: ER, extended-release; TEAE, Treatment Emergent Adverse Event. 


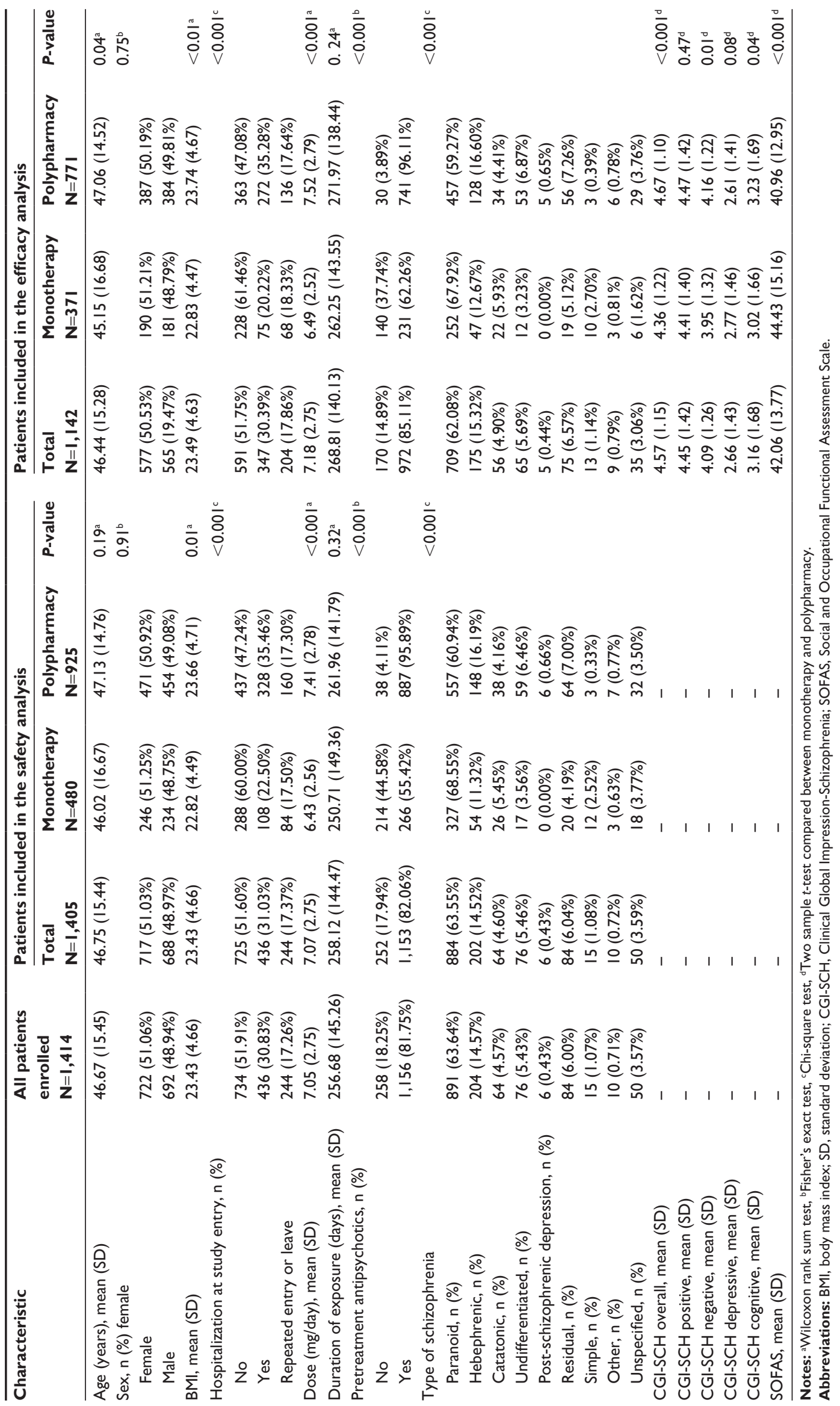


$7.05 \pm 2.75 \mathrm{mg}$. About $65.84 \%$ of patients in the safety analysis set were given other antipsychotics combined with PAL-ER (polypharmacy). The antipsychotics given in the polypharmacy group were risperidone $(n=328)$, olanzapine ( $n=234)$, quetiapine $(n=143)$, aripiprazole $(n=134)$, blonanserin $(n=109)$, perospirone $(n=33)$, clozapine $(n=1)$, and typical antipsychotics $(\mathrm{n}=520)$. Comparisons between monotherapy and polypharmacy groups demonstrated that the polypharmacy group had significantly higher body mass index (BMI) $(P=0.01)$, the rate of hospitalized patients $(P<0.001)$ and the rate of patients treated with pretreatment antipsychotics at the entry $(P<0.001)$, and mean daily dose of PAL-ER than monotherapy group $(P<0.001)$. The mean score of CGI-SCH overall, negative, and cognitive and SOFAS score at the baseline in the efficacy dataset were significantly worse in the polypharmacy group (overall: $P<0.001$; negative: $P=0.01$; cognitive: $P=0.04$; SOFAS: $P<0.001)$.

\section{Discontinuation rate}

The treatment discontinuation rates for any reason during the observation period are shown in Figure 2. Discontinuation rate was $34.66 \%$ during observation period. The withdrawals were due to patient's choice (12.60\%), due to AEs (10.32\%), the lack of efficacy $(8.75 \%)$, and other reasons $(10.75 \%)$. The post hoc analysis for monotherapy versus polypharmacy demonstrated a significantly higher discontinuation rate for any reason in the polypharmacy group $(37.10 \%)$ than in the monotherapy group $(29.40 \%$; log-rank test, $P=0.027)$. Chisquare tests revealed that reasons of discontinuations between both groups were not different $\left(\chi^{2}[3]=2.72, P=0.436\right)$; monotherapy versus polypharmacy were as follows: patient's choice: $11.67 \%$ versus $13.08 \%$, safety reasons: $8.13 \%$ versus $11.46 \%$, lack of efficacy: $6.88 \%$ versus $9.73 \%$, and other reasons: $14.79 \%$ versus $8.65 \%$.

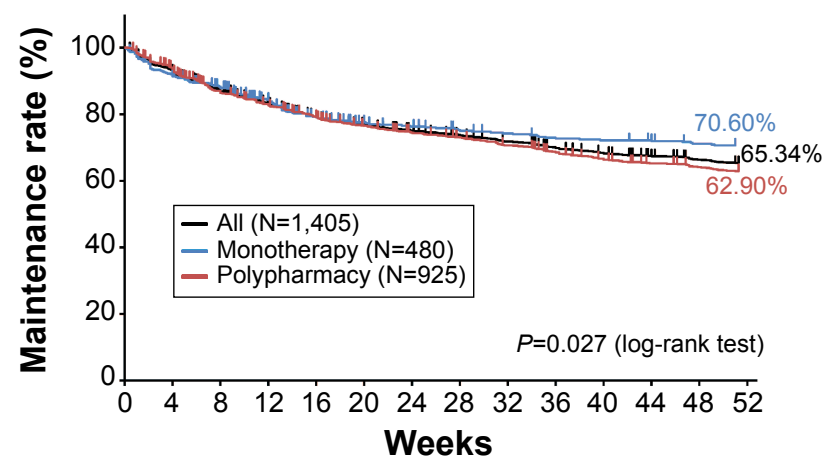

Figure 2 Kaplan-Meier estimate of time to discontinuation for any reason during the observation period (safety analysis set).

\section{Changes in social function and symptomatic improvement}

Time changes of the treatment in SOFAS and CGI-SCH scores, and results of post hoc $t$-tests using Bonferroni adjustment following GLMM are shown in Figures 3 and 4. One hundred and twelve patients were scored as "not enough information to make an evaluation" by physicians on SOFAS score and these data were handled as missing data. GLMM revealed a significant main effect for time in SOFAS ( $F[2$, $2,056]=23.25, P<0.001)$. The post hoc test revealed that there were significant improvements in the SOFAS score between the baseline and all follow-up points, and between 6 and 12 months. In addition to the main effect for time, main effect of monotherapy versus polypharmacy group was significant $(F[1,1,025]=32.18, P<0.001)$. No significant interaction was found between time and monotherapy versus polypharmacy $(F[2,2,056]=1.00, P=0.366)$. Regarding symptomatic scores, GLMM also revealed a significant main effect for time in all CGI scores (overall: $F[7,7,980]=85.54, P<0.001$; positive: $F[7,7,952]=91.20, P<0.001$; negative: $F[7,7,959]=69.71$, $P<0.001$; depressive: $F[7,7,945]=30.58, P<0.001$; cognitive: $F[7,7,924]=28.46, P<0.001)$. The post hoc test revealed that there were significant improvements between the baseline and each follow-up time point. For the post hoc comparisons of each adjacent time point, the CGI-SCH overall score, as well as the positive score were significantly improved over time from the baseline to 24 weeks and between 36 and 52 weeks. The CGI-SCH negative score was significantly improved over time from the baseline to 12 weeks, and between 36 and 52 weeks. The CGI-SCH depressive score was significantly improved over time from the baseline to 24 weeks. The CGI-SCH cognitive score was significantly improved over time from the baseline to 12 weeks. In addition to the main effect of time, main effect of monotherapy versus polypharmacy group was significant in all CGI scores (overall: $F[1,1,137]=162.29, P<0.001$; positive: $F[1,1,133]=135.50, P<0.001$; negative: $F[1,1,134]=221.54$, $P<0.001$; depressive: $F[1,1,132]=41.59, P<0.001$; cognitive: $F[1,1,129]=81.44, P<0.001)$. The interaction of time $\times$ monotherapy versus polypharmacy group was also significant in all CGI-SCH scores (overall: $F[7,7,98]=2.77$, $P=0.007$; positive: $F[7,7,952]=3.66, P<0.001$; negative: $F[7,7,959]=3.71, P<0.001$; depressive: $F[7,7,945]=3.50$, $P<0.001$; cognitive: $F[7,7,924]=1.30, P=0.247$ ).

\section{Factors associated with social functional remission}

All factors modeled and the results of the logistic regression analysis are showed in Table 2 . None of the variance inflation 


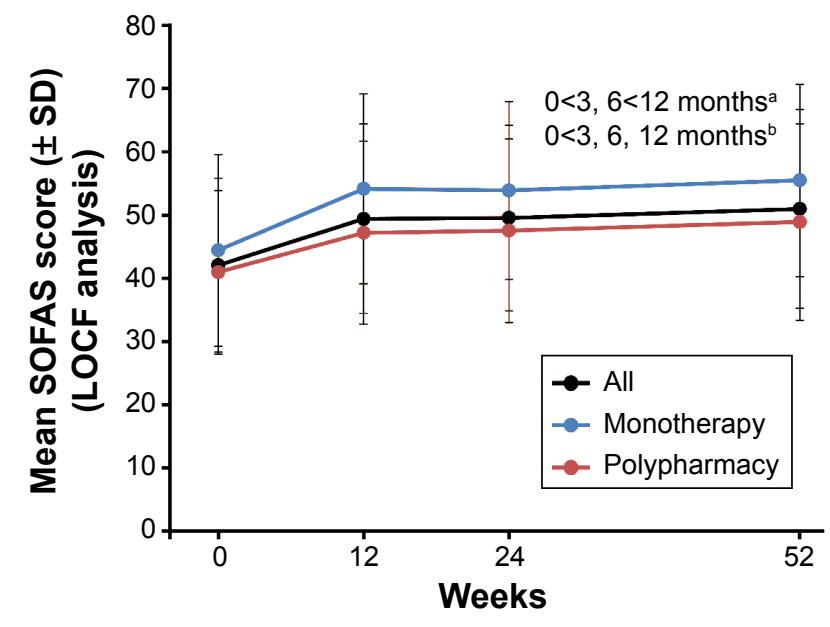

Figure 3 Mean SOFAS score over time during the observation period (LOCF analysis). Notes: Bars represent standard deviation (SD). Result of post hoc $t$-test with Bonferroni's adjustment after GLMM analysis $(P<0.05)$; ${ }^{\text {adifferences between each }}$ time point and ${ }^{b}$ changes from the baseline.

Abbreviations: SOFAS, Social and Occupational Functioning Assessment Scale; LOCF, last observation carried forward; GLMM, generalized linear mixed models. factor values were up to 7, indicating that multicollinearity in the following logistic regression model is not evident. Logistic regression analysis showed that sex $(P=0.048)$, baseline CGI negative score $(P=0.004)$, and $\Delta$ CGI positive at 4 weeks $(P=0.024)$ were significantly associated with the socially functional remission (Table 2). The percentage of patients showing social functional remission was $2.89 \%$ at the baseline and $13.22 \%$ at 52 weeks (the mean \pm SD duration of functional remission was $6.38 \pm 4.11$ months). Patients with $\geq 61$ points were $4.58 \%$ in the monotherapy group and $2.08 \%$ in the polypharmacy group at the baseline, and $15.90 \%$ in the monotherapy group and $11.93 \%$ in the polypharmacy group at 52 weeks. Finally, GLMM was used to examine the effect of interaction of time $\times$ monotherapy versus polypharmacy for the change of the percentage of social functional remission. While the effect of time was significant $(\mathrm{F}[1,615]=126.39, P<0.001)$ and the effect of
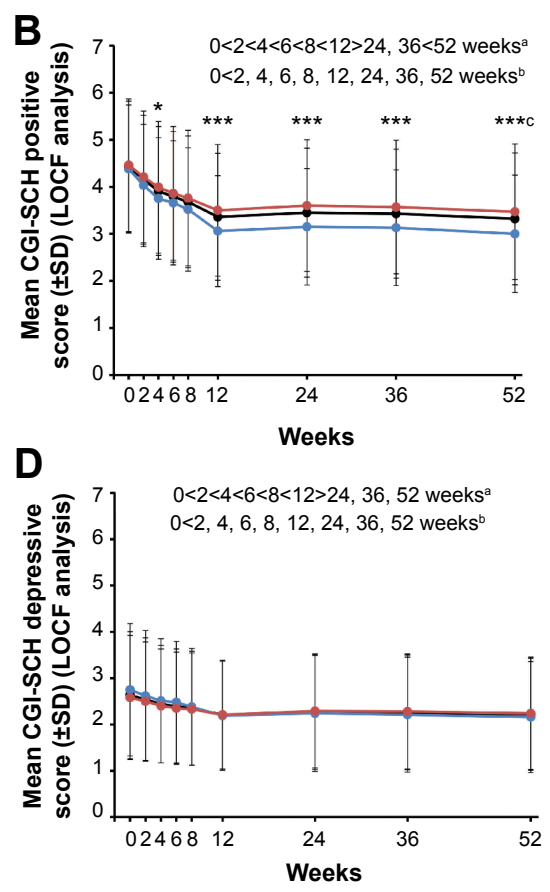

D

$0<2<4<6<8<12>24,36,52$ weeks $^{a}$

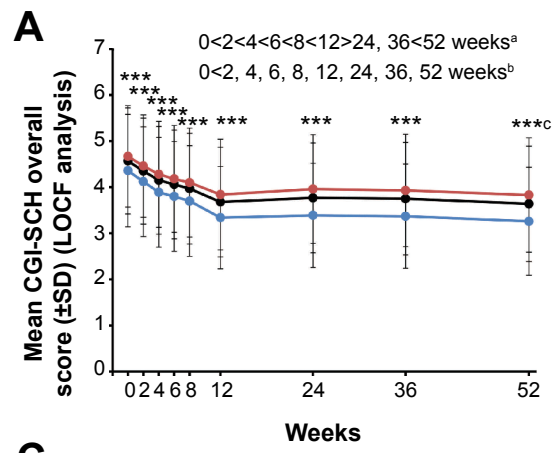

C

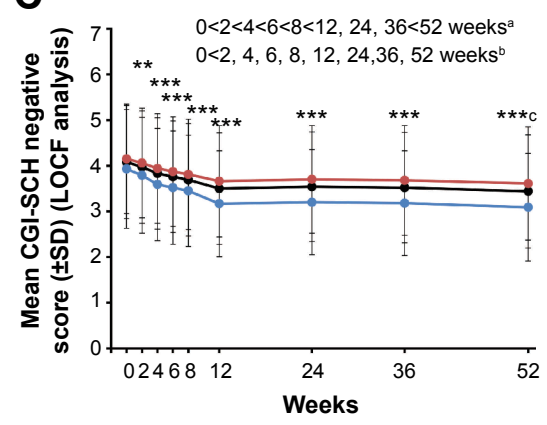

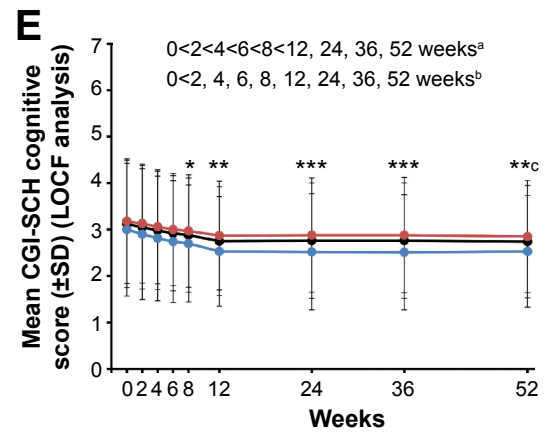

Figure 4 Mean CGI-SCH scores over time during the observation period (LOCF analysis).

Notes: (A) CGI-SCH overall, (B) CGI-SCH positive, (C) CGI-SCH negative, (D) CGI-SCH depressive, and (E) CGI-SCH cognitive score. Bars represent standard deviation

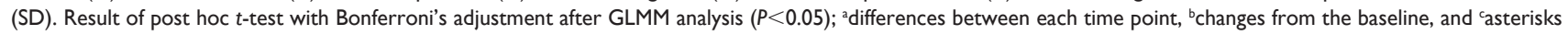
denote values that are significantly different between monotherapy and polypharmacy. $* P<0.05, * * P<0.01, * * * P<0.00$ I.

Abbreviations: CGI-SCH, Clinical Global Impression-Schizophrenia; LOCF, last observation carried forward; GLMM, generalized linear mixed models. 
Table 2 Logistic regression analysis of social function remission

\begin{tabular}{|c|c|c|c|c|c|c|}
\hline & B & SE & Wald & Odd ratios & $95 \% \mathrm{Cl}$ & $P$-value \\
\hline \multicolumn{7}{|l|}{ Demographics } \\
\hline Sex (male) & -0.60 & 0.30 & 3.89 & 0.55 & $0.30-1.00$ & $0.048^{*}$ \\
\hline Age & -0.02 & 0.01 & 1.99 & 0.98 & $0.96-1.01$ & 0.159 \\
\hline Polypharmacy & -0.57 & 0.34 & 2.82 & 0.56 & $0.29-1.10$ & 0.093 \\
\hline \multicolumn{7}{|l|}{ Baseline CGI-SCH score } \\
\hline Baseline CGI-SCH overall & -0.14 & 0.23 & 0.36 & 0.87 & $0.55-1.37$ & 0.546 \\
\hline Baseline CGI-SCH positive & -0.24 & 0.17 & 1.91 & 0.79 & $0.56-1.11$ & 0.167 \\
\hline Baseline CGI-SCH negative & -0.54 & 0.19 & 8.38 & 0.58 & $0.40-0.84$ & $0.004^{* *}$ \\
\hline Baseline CGI-SCH depressive & -0.04 & 0.15 & 0.07 & 0.96 & $0.72-1.29$ & 0.796 \\
\hline Baseline CGI-SCH cognitive & -0.05 & 0.13 & 0.11 & 0.96 & $0.74-1.24$ & 0.735 \\
\hline \multicolumn{7}{|l|}{$\Delta \mathrm{CGI}-\mathrm{SCH}$ score } \\
\hline$\Delta \mathrm{CGI}-\mathrm{SCH}$ overall at 2 weeks & -0.27 & 0.49 & 0.30 & 0.76 & $0.29-2.01$ & 0.587 \\
\hline$\Delta \mathrm{CGI}-\mathrm{SCH}$ positive at 2 weeks & -0.36 & 0.34 & 1.14 & 0.70 & $0.36-1.35$ & 0.285 \\
\hline$\Delta \mathrm{CGI}-\mathrm{SCH}$ negative at 2 weeks & 0.47 & 0.45 & 1.08 & 1.60 & $0.66-3.86$ & 0.300 \\
\hline$\Delta \mathrm{CGI}-\mathrm{SCH}$ depressive at 2 weeks & 0.22 & 0.45 & 0.24 & 1.25 & $0.5 I-3.02$ & 0.627 \\
\hline$\Delta \mathrm{CGI}-\mathrm{SCH}$ cognitive at 2 weeks & -0.34 & 0.54 & 0.40 & 0.71 & $0.25-2.06$ & 0.527 \\
\hline$\Delta \mathrm{CGI}-\mathrm{SCH}$ overall at 4 weeks & 0.10 & 0.43 & 0.06 & 1.11 & $0.48-2.59$ & 0.811 \\
\hline$\Delta \mathrm{CGI}-\mathrm{SCH}$ positive at 4 weeks & 0.74 & 0.33 & 5.12 & 2.10 & $1.10-3.99$ & $0.024 *$ \\
\hline$\Delta \mathrm{CGI}-\mathrm{SCH}$ negative at 4 weeks & -0.23 & 0.38 & 0.36 & 0.80 & $0.38-1.68$ & 0.550 \\
\hline$\Delta \mathrm{CGI}-\mathrm{SCH}$ depressive at 4 weeks & 0.49 & 0.40 & 1.47 & 1.63 & $0.74-3.60$ & 0.226 \\
\hline$\Delta \mathrm{CGI}-\mathrm{SCH}$ cognitive at 4 weeks & 0.76 & 0.42 & 3.17 & 2.13 & $0.93-4.89$ & 0.075 \\
\hline
\end{tabular}

Notes: $* P<0.05, * * P<0.01$.

Abbreviations: B, regression coefficient; SE, standard error; Cl, confidence interval; CGI-SCH, Clinical Global Impression-Schizophrenia.

monotherapy versus polypharmacy group was also significant $(\mathrm{F}[1,1,049]=14.54, P<0.001)$, interaction of time $\times$ group was not significant $(\mathrm{F}[1,615]=0.20, P=0.656)$.

\section{Safety}

A total of $33.52 \%$ of patients experienced at least one AE. Table 3 shows AEs occurring in greater than $1.00 \%$ of patients. The SAEs (serious AEs) occurring in greater than or equal to two cases and some frequent SAEs of antipsychotics in addition to the number of deaths are also listed in Table 3. The most commonly reported AEs were hyperprolactinemia (6.55\%), somnolence $(5.91 \%)$, malaise $(4.84 \%)$, increased weight $(2.85 \%)$, akathisia $(2.63 \%)$, insomnia $(2.56 \%)$, and extrapyramidal disorder (1.64\%). Post hoc analysis revealed significantly higher incidences of AEs in the polypharmacy group than those in the monotherapy group for all AEs $(P<0.001)$, psychiatric symptoms $(P=0.003)$, Parkinsonism ( $P=0.042)$, hyperprolactinemia $(P=0.002)$, increased blood prolactin level $(P=0.007)$, and increased weight $(P=0.003)$. Approximately, $8.75 \%$ of patients had one or more SAEs. Most commonly reported SAEs which occurred more than or equal to $1.00 \%$ were extrapyramidal disorder $(1.00 \%)$ and schizophrenia (1.00\%). One case $(0.07 \%)$ each of malignant neuroleptic syndrome and tardive dyskinesia was reported as a SAE. Post hoc analysis revealed significantly higher incidences of SAEs in the polypharmacy group than those in the monotherapy group, all SAEs $(P<0.001)$, psychiatric symptoms $(P=0.019)$, and Parkinsonism $(P=0.011)$. Twelve deaths $(0.85 \%)$ occurred during the surveillance. Causes of deaths were suicide $(n=3,0.21 \%)$, pneumonia $(n=2,0.14 \%)$, acute cardiac infarction $(\mathrm{n}=1,0.07 \%)$, pulmonary malignant neoplasm $(n=1,0.07 \%)$, sudden death $(n=1,0.07 \%)$, multiple organ failure $(\mathrm{n}=1,0.07 \%)$, cerebellar hemorrhage $(\mathrm{n}=1$, $0.07 \%)$, ileus $(n=1,0.07 \%)$, and unknown $(n=1,0.07 \%)$. Eleven of these patients were in the polypharmacy group $(0.21 \%)$ and one was in the monotherapy group $(1.19 \%)$. The incidence of death was not significantly different between both groups $(P=0.069)$.

\section{Discussion}

Data from this observational study demonstrated that patients treated with PAL-ER showed significant improvements in social function and symptoms and these can be controlled with a $34.66 \%$ discontinuation rate for any reason at 12 months. The discontinuation rate in the present study is generally consistent in magnitude to the rates observed in previous studies..$^{21,25-27}$ Since discontinuation for any reason is a global index of antipsychotic effectiveness and tolerability, the present result indicates that PAL-ER treatment is efficacious and well tolerated. ${ }^{25,26}$ Although scales measured in the 
Table 3 Adverse events (AEs) and serious AEs (safety analysis set)

\begin{tabular}{|c|c|c|c|c|c|c|c|}
\hline \multirow[t]{2}{*}{ Adverse drug reaction } & \multicolumn{2}{|c|}{$\begin{array}{l}\text { All } \\
N=1,405\end{array}$} & \multicolumn{2}{|c|}{$\begin{array}{l}\text { Monotherapy } \\
\mathrm{N}=\mathbf{4 8 0}\end{array}$} & \multicolumn{2}{|c|}{$\begin{array}{l}\text { Polypharmacy } \\
\mathrm{N}=925 \\
\end{array}$} & \multirow[t]{2}{*}{$P$-value } \\
\hline & $\mathbf{N}$ & (\%) & $\mathbf{N}$ & (\%) & $\mathbf{N}$ & (\%) & \\
\hline \multicolumn{8}{|l|}{$\geq 1 \% \mathrm{AEs}$} \\
\hline Total number of patients with adverse events & 471 & $(33.52)$ & 111 & $(23.13)$ & 360 & $(38.92)$ & $<0.001 * * *$ \\
\hline Insomnia & 36 & $(2.56)$ & 7 & $(1.46)$ & 29 & $(3.14)$ & 0.070 \\
\hline Schizophrenia & 18 & $(1.28)$ & 6 & $(1.25)$ & 12 & $(1.30)$ & 0.999 \\
\hline Psychiatric symptom & 20 & $(1.42)$ & 1 & $(0.21)$ & 19 & $(2.05)$ & $0.003^{* *}$ \\
\hline Akathisia & 37 & $(2.63)$ & 11 & $(2.29)$ & 26 & $(2.8 \mathrm{I})$ & 0.604 \\
\hline Extrapyramidal disorder & 23 & $(1.64)$ & 8 & $(1.67)$ & 15 & $(1.62)$ & 0.999 \\
\hline Parkinsonism & 22 & $(1.57)$ & 3 & $(0.63)$ & 19 & $(2.05)$ & $0.042 *$ \\
\hline Somnolence & 83 & $(5.91)$ & 24 & $(5.00)$ & 59 & $(6.38)$ & 0.341 \\
\hline Tremor & 15 & $(\mathrm{I} .07)$ & 3 & $(0.63)$ & 12 & $(1.30)$ & 0.288 \\
\hline Salivary hypersecretion & 15 & $(1.07)$ & 4 & $(0.83)$ & 11 & $(1.19)$ & 0.785 \\
\hline Malaise & 68 & $(4.84)$ & 25 & $(5.2 I)$ & 43 & $(4.65)$ & 0.694 \\
\hline Hyperprolactinemia & 92 & $(6.55)$ & 18 & $(3.75)$ & 74 & $(8.00)$ & $0.002 * *$ \\
\hline Blood prolactin increased & 23 & $(1.64)$ & 2 & $(0.42)$ & 21 & $(2.27)$ & $0.007^{* *}$ \\
\hline Weight increased & 40 & $(2.85)$ & 5 & $(1.04)$ & 35 & $(3.78)$ & $0.003 * *$ \\
\hline \multicolumn{8}{|l|}{ SAEs } \\
\hline Total number patients with serious adverse events & 123 & $(8.75)$ & 22 & $(4.58)$ & 101 & $(10.92)$ & $<0.00 I^{* * *}$ \\
\hline Pneumonia & 3 & $(0.21)$ & 0 & $(0.00)$ & 3 & $(0.32)$ & 0.555 \\
\hline Hyperprolactinemia & 6 & $(0.43)$ & 2 & $(0.42)$ & 4 & $(0.43)$ & 0.999 \\
\hline Polydipsia & 2 & $(0.14)$ & 0 & $(0.00)$ & 2 & $(0.22)$ & 0.550 \\
\hline Anxiety & 3 & $(0.21)$ & 0 & $(0.00)$ & 3 & $(0.32)$ & 0.555 \\
\hline Completed suicide & 3 & $(0.21)$ & 0 & $(0.00)$ & 3 & $(0.32)$ & 0.555 \\
\hline Delusion & 3 & $(0.21)$ & 0 & $(0.00)$ & 3 & $(0.32)$ & 0.555 \\
\hline Hallucination & 3 & $(0.21)$ & 0 & $(0.00)$ & 3 & $(0.32)$ & 0.555 \\
\hline Hallucination, auditory & 7 & $(0.50)$ & 0 & $(0.00)$ & 7 & $(0.76)$ & 0.103 \\
\hline Irritability & 5 & $(0.36)$ & 1 & $(0.21)$ & 4 & $(0.43)$ & 0.666 \\
\hline Paranoia & 2 & $(0.14)$ & 0 & $(0.00)$ & 2 & $(0.22)$ & 0.550 \\
\hline Schizophrenia & 14 & $(1.00)$ & 6 & $(1.25)$ & 8 & $(0.86)$ & 0.573 \\
\hline Schizophrenia, paranoid type & 4 & $(0.28)$ & 0 & $(0.00)$ & 4 & $(0.43)$ & 0.306 \\
\hline Psychiatric symptom & 10 & $(0.7 I)$ & 0 & $(0.00)$ & 10 & $(1.08)$ & $0.019 *$ \\
\hline Dystonia & 4 & $(0.28)$ & I & $(0.21)$ & 3 & $(0.32)$ & 0.999 \\
\hline Extrapyramidal disorder & 14 & $(1.00)$ & 4 & $(0.83)$ & 10 & $(1.08)$ & 0.782 \\
\hline Neuroleptic malignant syndrome & 1 & $(0.07)$ & 0 & $(0.00)$ & 1 & $(0.11)$ & 0.999 \\
\hline Parkinsonism & 12 & $(0.85)$ & 0 & $(0.00)$ & 12 & $(1.30)$ & $0.011 *$ \\
\hline Tardive dyskinesia & 1 & $(0.07)$ & 0 & $(0.00)$ & 1 & $(0.11)$ & 0.999 \\
\hline Pneumonia aspiration & 3 & $(0.21)$ & 2 & $(0.42)$ & I & $(0.11)$ & 0.270 \\
\hline Intestinal obstruction & 3 & $(0.21)$ & I & $(0.21)$ & 2 & $(0.22)$ & 0.342 \\
\hline Blood glucose increased & 2 & $(0.14)$ & 0 & $(0.00)$ & 2 & $(0.22)$ & 0.550 \\
\hline Blood prolactin increased & 2 & $(0.14)$ & 1 & $(0.21)$ & 1 & $(0.11)$ & 0.999 \\
\hline Femur fracture & 2 & $(0.14)$ & I & $(0.21)$ & I & $(0.11)$ & 0.999 \\
\hline Total number of deaths & 12 & $(0.85)$ & I & $(0.21)$ & 11 & $(1.19)$ & 0.069 \\
\hline
\end{tabular}

Notes: $* P<0.05, * * P<0.01$, ***P $<0.001$. All AEs occurring in $\geq 1 \%$ of patients and serious AEs (SAEs) occurring more than one time except for some frequent $S A E s$ of antipsychotics are shown in this table.

present study are different from those of previous randomized controlled pivotal trials, observed findings of improvements on both symptoms and social function are concordant with randomized controlled trials and a pooled data analysis that have demonstrated a significant improvement of personal and social functioning following treatment with PAL-ER. ${ }^{18-21}$ A significant increase in the rate of patients with social functional remission was also observed. The data clearly indicate the clinical effectiveness of PAL-ER in terms of improving social functioning in patients with schizophrenia. Improving social functioning is an essential component of optimizing long-term outcomes for patients with schizophrenia. Even with good symptom control, poor personal and social functioning should compromise the potential of patients to optimize their ability to function in daily life. Therefore, the impact of antipsychotic treatment on functioning is the most important factor of improving the long-term prognosis of patients with schizophrenia. ${ }^{7}$ 
Regarding improvements of symptoms of schizophrenia, improvements were observed within the first 2 weeks of the treatment and improved symptoms were maintained during the observational period. This is consistent with the results of previous trials of PAL-ER. Recent data have demonstrated the patient's likelihood to "respond" to a given antipsychotic medication as symptom reduction is evident within the first 2 weeks of starting an antipsychotic medication. ${ }^{15-17}$ Early response to antipsychotic drug therapy has been associated subsequently with an increased likelihood of achieving symptom remission, with greater improvement on functional outcomes. ${ }^{15} \mathrm{We}$ postulate that early symptomatic improvement caused by PAL-ER should be associated with subsequent improvement on social function, and early symptomatic improvement suggests the clinical usefulness of PAL-ER in patients with schizophrenia.

Results from logistic regression analysis demonstrate the improvements in schizophrenic symptoms associated with PAL-ER treatment; early improvement in positive symptoms was associated with social functional remission. Previous studies have also demonstrated strong associations between good functional outcomes and symptomatic improvements. ${ }^{7,10,11}$ They also reported that a lower Positive and Negative Symptom Scale negative score was predictive of functional remission. Several studies have implicated negative symptoms and neurocognitive deficits in poor functional outcomes. ${ }^{25}$ Although the present study did not show a relationship between improvements in negative symptoms caused by treatment and socially functional remission, a significant association of severity of negative symptoms at baseline was observed. Our results are in line with those of previous studies ${ }^{28,29}$ that support the accepted notion about the relationship between negative symptoms and social functional outcomes in patients with schizophrenia. At the same time, however, our results demonstrate that there are still important limitations to current treatments for schizophrenia. First, only approximately $20 \%$ of patients had social functional remission in the present study. Second, severity of negative symptoms at baseline had marked effects on social functional outcomes. Currently available treatments for negative symptoms appear to have modest benefits, with the result that negative symptoms continue to limit patient recovery. ${ }^{28,29}$ Despite the advent of antipsychotics, the relatively low rate of social functional remission observed in the present study demonstrates that there are still important limitations to current treatments for chronic schizophrenia. More effective novel drugs specifically targeting reducing negative symptoms are needed to improve the prognosis of patients. As with symptomatology, sex was also highly associated with social functional remission. It is well known that women with schizophrenia showed better prognosis and social function than men with schizophrenia. ${ }^{30}$ Several sociodemographic factors such as status of employment, and a better premorbid social adjustment, have been reported as potential predictors to be highly predictive of social functional remission as well as symptomatic improvement. ${ }^{10,12}$ In this study, we did not collect these important factors related to social functional remission. This is also one of the limitations of the present study.

Although we recommended monotherapy with PAL-ER, more than $60 \%$ of patients were treated with antipsychotic polypharmacy (PAL-ER combined with other antipsychotics). In Japan, polypharmacy with high-dose antipsychotics has been common. A previous study also reported that more than $65 \%$ patients with schizophrenia were given antipsychotic polypharmacy. ${ }^{31}$ The high rate of polypharmacy in the study is a mirror of current prescription profiles of pharmacotherapy for schizophrenia patients in Japan. Despite a lack of evidence of increased efficacy and a higher risk for adverse effects related to polypharmacy, it has become more common since the development of second-generation antipsychotics. ${ }^{31,32}$ In this study, PAL-ER monotherapy group showed more improvement in symptoms and a lower discontinuation rate than the polypharmacy group. The results may suggest clinical advantages of monotherapy over polypharmacy. Contrary to the present result, a previous study documented the lower discontinuation rate in the polypharmacy group than that in the PAL-ER monotherapy group. ${ }^{32}$ The present study is an observational study; therefore, causality between polypharmacy and efficacy outcomes remains to be clarified. For example, it would be possible that patients with polypharmacy would have a more severe form of schizophrenia, and consequently physicians combine antipsychotics. It is one of the limitations of the present study. On the other hand, the improvement of social functioning and the rate of social functional remission did not differ between the groups. The results also demonstrated that whether patients were given monotherapy or polypharmacy was not related to the social functional outcome. These suggest that PAL-ER treatment should improve social functioning regardless of monotherapy or polypharmacy, given that improvement in both groups was clinically relevant.

The safety data in the present study are generally concordant with the safety profile of PAL-ER documented in previous studies, with no unexpected findings from long-term treatment and a safety profile consistent with the 
known pharmacological profile of PAL. The mean dose of PAL-ER in the polypharmacy group was higher than that in the PAL-ER monotherapy group. Furthermore, a considerable number of patients with polypharmacy were given other D2 antagonists in addition to PAL-ER. The different safety profile of monotherapy versus polypharmacy might be associated with high-dose antipsychotic therapy in the polypharmacy group. Because this study was a naturalistic one with flexible dose treatment, we did not evaluate the relationship between mean dose of PAL-ER and increasing risks of AEs in the monotherapy group. This is one of limitations of the study.

\section{Conclusion}

This 1-year observational study data from 1,405 patients with schizophrenia indicates that effective symptom control and improvement in social functioning as assessed by SOFAS can be maintained with PAL-ER. Treatment with PAL-ER was generally safe and well tolerated. The findings expand on the evidence obtained from randomized controlled clinical trials with PAL-ER and support previous positive findings of social functional improvement. Furthermore, the data suggest that early response to antipsychotic treatment may result in better outcomes for consequent social functional outcomes.

\section{Acknowledgments}

This study is registered at umin.ac.jp (Identifier: UMIN00001559933). This study was funded by Janssen Pharmaceutical K.K., a division of Johnson \& Johnson in Japan.

\section{Author contributions}

All authors contributed to interpretation of the results, developed the draft of the manuscript, participated in subsequent revisions, and read and approved the final manuscript.

\section{Disclosure}

All authors disclose that they are full-time employees of Janssen Pharmaceutical K.K., a division of Johnson \& Johnson in Japan. The authors report no other conflicts of interest.

\section{References}

1. European Medicines Agency. Guideline on clinical investigation of medicinal products, including depot preparations in the treatment of schizophrenia. Available from: http://www.ema.europa.eu/docs/en_GB/ document_library/Scientific_guideline/2012/10/WC500133437.pdf. Accessed January 22, 2015.

2. Lehman AF. Developing an outcomes-oriented approach for the treatment of schizophrenia. J Clin Psychiatry. 1999;60(Suppl 19):S30-S35.

3. Andreasen NC, Carpenter WT Jr, Kane JM, Lasser RA, Marder SR, Weinberger DR. Remission in schizophrenia: proposed criteria and rationale for consensus. Am J Psychiatry. 2005;162(3):441-449.
4. Nasrallah HA, Targum SD, Tandon R, et al. Defining and measuring clinical effectiveness in the treatment of schizophrenia. Psychiatr Serv. 2005;56:273-282.

5. Yeomans D, Taylor M, Currie A, et al. Resolution and remission in schizophrenia: getting well and staying well. Adv Psychiatr Treat. 2010; 16:86-95.

6. Lieberman JA, Malaspina D, Jarskog LF. Preventing clinical deterioration in the course of schizophrenia: the potential for neuroprotection. CNS Spectr. 2006;11(Suppl 4):1-13.

7. Larnbert M, Naber D. Current issues in schizophrenia: overview of patient acceptability, functioning capacity and quality of life. CNS Drugs. 2004;18:1-13.

8. Haro JM, Novick D, Suarez D, et al. Predictors of the course of illness in outpatients with schizophrenia: a prospective three year study. Prog Neuropsychopharmacol Biol Psychiatry. 2008;32:1287-1292.

9. Lambert M, Schimmelmann BG, Naber D, et al. Prediction of remission as a combination of symptomatic and functional remission and adequate subjective wellbeing in 2,960 patients with schizophrenia. J Clin Psychiatry. 2006;67:1690-1697.

10. Spellmann I, Riedel M, Schennach R, et al. One-year functional outcomes of naturalistically treated patients with schizophrenia. Psychiatry Res. 2012;198(3):378-385.

11. Schennach-Wolff R, Jäger M, Seemüller F, et al. Defining and predicting functional outcome in schizophrenia and schizophrenia spectrum disorders. Schizophr Res. 2009;113:210-217.

12. Lambert M, Naber D, Schacht A, et al. Rates and predictors of remission and recovery during 3 years in 392 never-treated patients with schizophrenia. Acta Psychiatr Scand. 2008;118(3):220-229.

13. Mortimer AM. Symptom rating scales and outcome in schizophrenia. Br J Psychiatry Suppl. 2007;50:S7-S14.

14. Lipkovich IA, Deberdt W, Csernansky JG, et al. Defining "good" and "poor" outcomes in patients with schizophrenia or schizoaffective disorder: a multidimensional data-driven approach. Psychiatry Res. 2009; 170:161-167.

15. Ascher-Svanum H, Nyhuis AW, Faries DE, et al. Clinical, functional and economic ramifications of early nonresponse to antipsychotics in the naturalistic treatment of schizophrenia. Schizophr Bull. 2008;34(6): 1163-1171.

16. Kinon BJ, Chen L, Ascher-Svanum H, et al. Early response to antipsychotic drug therapy as a clinical marker of subsequent response in the treatment of schizophrenia. Neuropsychopharmacology. 2010; 35:581-590.

17. Leucht S, Shamsi SA, Busch R, et al. Predicting antipsychotic drug response - replication and extension to six weeks in an international olanzapine study. Schizophr Res. 2008;101(1-3):312-319.

18. Davidson M, Emsley R, Kramer M, et al. Efficacy, safety and response of paliperidone extended-release tablets (paliperidone ER): results of a 6-week, randomized, placebo-controlled study. Schizophr Res. 2007;93: $117-130$.

19. Kane J, Canas F, Kramer M, et al. Treatment of schizophrenia with paliperidone extended-release tablets: a 6-week placebo-controlled trial. Schizophr Res. 2007;90:147-161.

20. Kramer M, Simpson G, Maciulis V, et al. Paliperidone extended-release tablets for prevention of symptom recurrence in patients with schizophrenia: a randomized, double-blind, placebo-controlled study. J Clin Psychopharmacol. 2007;27:6-14.

21. Emsley R, Berwaerts J, Eerdekens M, et al. Efficacy and safety of oral paliperidone extended-release tablets in the treatment of schizophrenia: pooled data from three 52-week open-label studies. Int Clin Psychopharmacol. 2008;23:343-356.

22. Hough D, Nuamah IF, Lim P, et al. Independent effect of paliperidone extended release on social functioning beyond its effect on positive and negative symptoms of schizophrenia A Mediation Analysis. J Clin Psychopharmacol. 2009;29(5):496-497.

23. American Psychiatric Association. Diagnostic and Statistical Manual of Mental Disorders, Fourth Edition, Text Revision, DSM-IV-TR. Washington, DC: American Psychiatric Association; 2000. 
24. Haro JM, Kamath SA, Ochoa S, et al. The Clinical Global ImpressionSchizophrenia scale: a simple instrument to measure the diversity of symptoms present in schizophrenia. Acta Psychiatr Scand Suppl. 2003;416:16-23.

25. Lieberman JA, Stroup TS, McEvoy JP, et al. Effectiveness of antipsychotic drugs in patients with chronic schizophrenia. NEngl J Med. 2005; 353(12):1209-1223.

26. Haro JM, Suarez D, Novick D, et al. Three-year antipsychotic effectiveness in the outpatient care of schizophrenia: observational versus randomized studies results. Eur Neuropsychopharmacol. 2007; 17:235-244

27. Schreiner A, Hargarter L, Hitschfield K, et al. Clinical effectiveness and resource utilization of paliperidone ER for schizophrenia: Pharmacoepidemiologic International Longitudinal Antipsychotic Registry (PILAR). Curr Med Res Opin. 2014;30(7):1279-1289.

28. Tamminga CA, Buchanan RW, Gold JM. The role of negative symptoms and cognitive dysfunction in schizophrenia outcome. Int Clin Psychopharmacol. 1998;13 (Suppl 3):S21-S26.
29. Greenwood KE, Landau S, Wykes T. Negative symptoms and specific cognitive impairments as combined targets for improved functional outcome within cognitive remediation therapy. Schizophr Bull. 2005; 31(4):910-921.

30. Usall J, Haro JM, Araya S, et al. Social functioning in schizophrenia: what is the influence of gender? Eur J Psychiat. 2007;21:199-205.

31. Yoshio T. Prescription profiles for pharmacological treatment of Japanese inpatients with schizophrenia: comparison between 2007 and 2009. Hum Psychopharmacol. 2012;27:70-75.

32. Correll CU, Rummel-Kluge C, Corves C, Kane JM, Leucht S. Antipsychotic combinations vs monotherapy in schizophrenia: a meta-analysis of randomized controlled trials. Schizophr Bull. 2009;35(2):443-457.

33. Janssen Pharmaceutical K.K., Japan. Special Drug Use-results Survey for Long-term Administration of Invega $3 \mathrm{mg} / 6 \mathrm{mg} / 9 \mathrm{mg}$ Tablet. Available from: https://upload.umin.ac.jp/cgi-open-bin/ctr/ctr.cgi?function= brows\&action=brows \& type $=$ summary \& recptno=R000018126\&langu age $=$ E. Identifier: UMIN000015599. Accessed January 22, 2015.
Neuropsychiatric Disease and Treatment

\section{Publish your work in this journal}

Neuropsychiatric Disease and Treatment is an international, peerreviewed journal of clinical therapeutics and pharmacology focusing on concise rapid reporting of clinical or pre-clinical studies on a range of neuropsychiatric and neurological disorders. This journal is indexed on PubMed Central, the 'PsycINFO' database and CAS,

\section{Dovepress}

and is the official journal of The International Neuropsychiatric Association (INA). The manuscript management system is completely online and includes a very quick and fair peer-review system, which is all easy to use. Visit http://www.dovepress.com/testimonials.php to read real quotes from published authors.

Submit your manuscript here: http://www.dovepress.com/neuropsychiatric-disease-and-treatment-journal 